\title{
Influence of heme $c$ attachment on heme conformation and potential
}

\author{
Jesse G. Kleingardner ${ }^{1,2}$ - Benjamin D. Levin ${ }^{3}$. Giorgio Zoppellaro ${ }^{4} \cdot \mathrm{K}_{\text {. Kristoffer Andersson }}^{5} \cdot$ Sean J. Elliott ${ }^{3}$. \\ Kara L. Bren ${ }^{1}$
}

Received: 10 May 2018 / Accepted: 16 August 2018

(c) SBIC 2018

\begin{abstract}
Heme $c$ is characterized by its covalent attachment to a polypeptide. The attachment is typically to a CXXCH motif in which the two Cys form thioether bonds with the heme, "X" can be any amino acid other than Cys, and the His serves as a heme axial ligand. Some cytochromes $c$, however, contain heme attachment motifs with three or four intervening residues in a $\mathrm{CX}_{3} \mathrm{CH}$ or $\mathrm{CX}_{4} \mathrm{CH}$ motif. Here, the impacts of these variations in the heme attachment motif on heme ruffling and electronic structure are investigated by spectroscopically characterizing $\mathrm{CX}_{3} \mathrm{CH}$ and $\mathrm{CX}_{4} \mathrm{CH}$ variants of Hydrogenobacter thermophilus cytochrome $c_{552}$. In addition, a novel $\mathrm{CXCH}$ variant is studied. ${ }^{1} \mathrm{H}$ and ${ }^{13} \mathrm{C} \mathrm{NMR}, \mathrm{EPR}$, and resonance Raman spectra of the protein variants are analyzed to deduce the extent of ruffling using previously reported relationships between these spectral data and heme ruffling. In addition, the reduction potentials of these protein variants are measured using protein film voltammetry. The $\mathrm{CXCH}$ and $\mathrm{CX}_{4} \mathrm{CH}$ variants are found to have enhanced heme ruffling and lower reduction potentials. Implications of these results for the use of these noncanonical motifs in nature, and for the engineering of novel heme peptide structures, are discussed.
\end{abstract}

Keywords Heme distortion $\cdot$ Heme ruffling $\cdot$ Reduction potential $\cdot$ Cytochrome $c \cdot$ Cytochrome $c$ maturation

\section{Introduction}

Heme $c$ is a cofactor that is widely distributed in nature [1]. Most frequently, its function is electron transfer, but heme $c$ also serves as the active site of enzymes such as cytochrome (cyt) $c$ nitrite reductase [2], bacterial peroxidases [3, 4], and $\mathrm{MauG}$, which plays a role in tryptophan tryptophylquinone

Electronic supplementary material The online version of this article (https://doi.org/10.1007/s00775-018-1603-3) contains supplementary material, which is available to authorized users.

Kara L. Bren

bren@chem.rochester.edu

1 Department of Chemistry, University of Rochester, Rochester, NY 14627-0216, USA

2 Department of Chemistry and Biochemistry, Messiah College, Mechanicsburg, PA 17055, USA

3 Department of Chemistry, Boston University, Boston, MA 02215-2521, USA

4 Regional Center of Advanced Technologies and Materials, 17. listopadu 12, 77146 Olomouc, Czech Republic

5 Department of Biosciences, University of Oslo, PO Box 1066, Blindern, 0316 Oslo, Norway biogenesis [5]. In addition, mitochondrial cyt $c$ performs both electron transfer in respiration and peroxide-mediated lipid oxidation in apoptosis [6].

Heme $c$ is formed by the covalent attachment of iron protoporphyrin IX (heme $b$ ) to two Cys residues in the polypeptide in a process assisted by maturation factors. Nature has evolved a number of different pathways for this process, suggesting that heme $c$ is required by most organisms [7-11]. The mechanism of heme $c$ biosynthesis and the functional relevance of heme $c$ covalent attachment have attracted considerable attention [10,12-15]. One consequence of heme covalent attachment is the enhancement of heme ruffling, which is the out-of-plane distortion in which the heme pyrroles twist in alternating directions moving around the porphyrin (Fig. 1) [16, 17]. In cyts $c$ and other heme proteins, heme ruffling is proposed to modulate reduction potential, with increased ruffling lowering potential [18-21]. Furthermore, variations in ruffling are proposed to impact the electronic coupling to redox partners, with increased ruffling decreasing coupling to donors or acceptors at the heme edge [22-25]. The structure of the heme attachment peptide, which is usually comprised of a $\mathrm{CXXCH}$ sequence, has been identified as a key determinant of ruffling [17, 19, 26, 27]. 
A
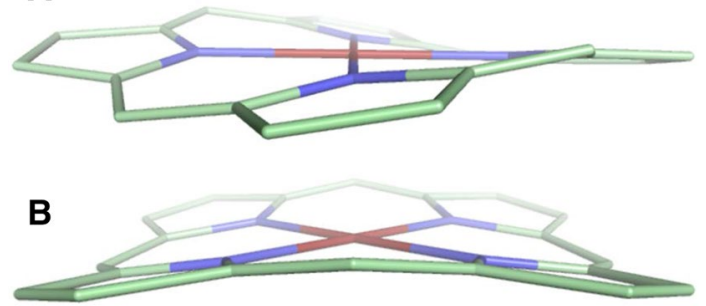

Fig. 1 Ruffled heme shown from two angles: a looking down the Fe$\mathrm{N}$ (pyrrole) bond, $\mathbf{b}$ centered on a bridging meso carbon. The Fe porphyrin model has a ruffling distortion of $1.0 \AA$. The structures were generated using Cartesian coordinates of a symmetric, planar iron porphyrin with typical bond lengths and angles. An idealized 1.0- $\AA$ ruffling distortion was applied using a quantitative description of the ruffling normal mode previously described, where the square root of the sum of the out-of-plane displacements of the core porphyrin atoms is equal to $1.0 \AA$ [16]. Reprinted with permission from Kleingardner and Bren [15], Copyright 2015 American Chemical Society

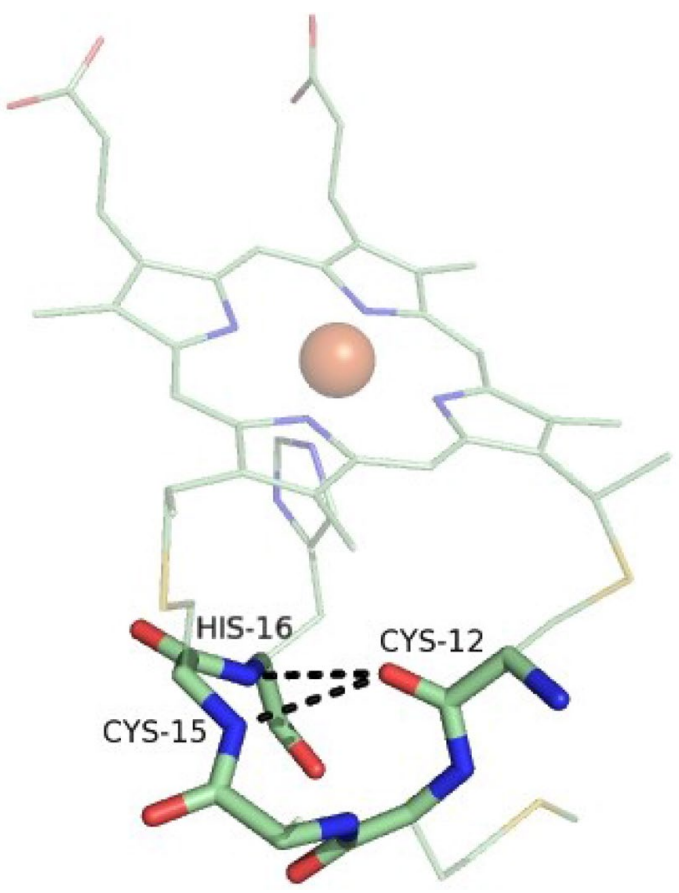

Fig. 2 Heme attached to a $\mathrm{CXXCH}$ heme attachment motif peptide from the structure of $H t$ cyt $c_{552}$ [28] with the backbone of the peptide highlighted. Hydrogen bonds are shown between the backbone carbonyl of Cys 12 and the backbone amide of Cys15, as well as between the backbone carbonyl of Cys12 and the backbone amide of His16. These hydrogen bonds are proposed to stabilize the ruffled heme conformation that is largely conserved in hemes $c$ [19]

Figure 2 highlights the backbone of the $\mathrm{CXXCH}$ motif, the structure of which has been implicated in stabilizing ruffled heme [19].

A small number of cyts $c$ have more than two residues between the two Cys that are attached to heme. In the tetraheme cyts $c_{3}$, two hemes are attached to canonical $\mathrm{CXXCH}$ motifs, while two are attached to $\mathrm{CX}_{4} \mathrm{CH}$ motifs. In structural studies, the hemes with the extended attachment motifs were found to be more ruffled than those bound to the canonical motifs, and the heme attachment motif has been implicated in these variations of ruffling [27, 29-31]. These reports inspired this study in which we investigate the influence of the length of the heme $c$ attachment motif on heme ruffling, electronic structure, and reduction potential. As a model system, we make use of Hydrogenobacter thermophilus ( $H t)$ cyt $c_{552}$, a small, monoheme, thermostable bacterial cyt $c$ with His/Met axial ligation. $H t$ cyt $c_{552}$ is a convenient model system for this study, because it is structurally characterized $[28,32]$ and has proven amenable to the introduction of a wide range of mutations, including some known to impact heme conformation [19-21]. In an investigation of the substrate specificity of cyt $c$ maturation systems, we expressed $H t$ cyt $c_{552}$ variants with three or four residues between the two Cys as well as a variant with a novel contracted heme attachment motif (CGCH) [33]. Here, we characterize the heme conformation in these variants using spectroscopic methods and determine the effects of nonplanar distortion on electronic structure and reduction potential. The results inform on the effects of the heme attachment motif on heme conformation, as well as implications of these effects for function. Furthermore, understanding how the heme attachment motif impacts heme conformation lays the groundwork for the production of novel heme peptides (microperoxidases) with different structural and electronic properties for a range of applications [34-36].

\section{Experimental section}

\section{Protein preparation}

Site-directed mutagenesis, expression, and purification of $H t$ cyt $c_{552}$ were performed as detailed in a prior study [33]. Briefly, the protein variants were expressed in LB medium to obtain natural isotope abundance samples, and purified as described [33]. For isotopic labeling, proteins were expressed in minimal (M9) medium supplemented with ${ }^{15} \mathrm{NH}_{4} \mathrm{Cl}$ for uniform ${ }^{15} \mathrm{~N}$ labeling or ${ }^{13} \mathrm{C}$-labeled 5-aminolevulinic acid (ALA) for ${ }^{13} \mathrm{C}$-labeling of heme core carbons as detailed elsewhere [20,37]. ALA derivatives with two different isotope labels were used, $4-{ }^{13} \mathrm{C}$-ALA and $5-{ }^{13} \mathrm{C}$ ALA, the syntheses of which are described in Supplementary Material. Resultant patterns of heme ${ }^{13} \mathrm{C}$ labeling are shown in Fig. 3. 


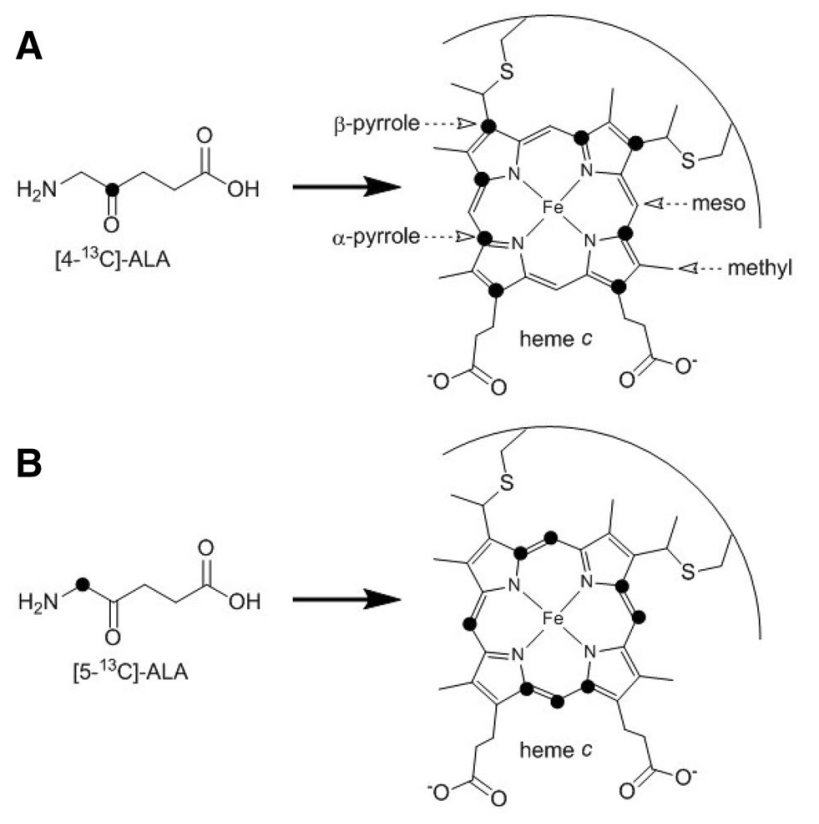

Fig. 3 Isotope-labeling patterns of heme obtained by incorporation of either a $4-{ }^{13} \mathrm{C}$-ALA or b $5-{ }^{13} \mathrm{C}$-ALA. Reprinted with permission from Kleingardner et al. [40], Copyright 2013 American Chemical Society

\section{Electrochemistry}

Protein film voltammetry (PFV) experiments conducted as reported previously [19, 38]. Briefly, an Ecochemie PGSTAT30 electrochemical analyzer was used, along with a water-jacketed cell in a three-electrode configuration. A standard calomel electrode (SCE) reference was maintained at room temperature throughout each experiment. All potentials were converted and reported versus the standard hydrogen electrode (SHE). Polycrystalline gold wire fixed in epoxy resin served as working electrodes. The gold wire was first prepared by polishing with a diamond suspension followed by successively finer alumina $(1.0,0.3$, and $0.05 \mu \mathrm{m})$. Final cleaning for each electrode was performed electrochemically by cycling between 0.2 and $1.35 \mathrm{~V}$ in $0.1 \mathrm{M} \mathrm{H}_{2} \mathrm{SO}_{4}$. Cleaned electrodes were modified in a $1 \mathrm{mM}$ ethanolic 6-mercaptohexanol solution for $12 \mathrm{~h}$. After rinsing with ethanol and water, electrodes were considered ready for use.

A typical experiment was carried out on the bench top with a mixed buffer: $50 \mathrm{mM}$ citrate $/ 50 \mathrm{mM}$ potassium phosphate at $0{ }^{\circ} \mathrm{C}$. Buffers were degassed with either argon or nitrogen prior to each measurement. Protein film was generated by applying a $2-\mu \mathrm{L}$ aliquot directly to the surface. Excess sample was washed away with buffer. Voltammograms were collected at $200 \mathrm{mV} \mathrm{s}^{-1}$ and cycled until a stable signal was observed. Data analysis was carried out by subtracting the non-Faradaic current using SOAS [39].

\section{NMR spectroscopy}

Oxidized $\left(\mathrm{Fe}^{\mathrm{III}}\right)$ and reduced $\left(\mathrm{Fe}^{\mathrm{II}}\right)$ protein samples at protein concentrations of $0.5-2.0 \mathrm{mM}$ were prepared as described in prior work [40]. 1-D ${ }^{1} \mathrm{H}-\mathrm{NMR}, 1-\mathrm{D}{ }^{13} \mathrm{C}-\mathrm{NMR}, 2-\mathrm{D}{ }^{1} \mathrm{H}-$ ${ }^{13} \mathrm{C}$ heteronuclear multiple quantum coherence (HMQC), and 2-D ${ }^{1} \mathrm{H}^{-}{ }^{15} \mathrm{~N}$ heteronuclear single quantum coherence (HSQC) spectra were obtained on $\mathrm{Fe}^{\mathrm{III}}$ samples as described [40] using a 500-MHz Varian Inova spectrometer equipped with a triple-resonance probe. In addition, a ${ }^{1} \mathrm{H}-{ }^{15} \mathrm{~N}$ HSQC spectrum was obtained for each uniformly ${ }^{15} \mathrm{~N}$-labeled sample in the diamagnetic $\mathrm{Fe}^{\mathrm{II}}$ oxidation state. NMR spectra were referenced indirectly to 4,4-dimethyl-4-silapentane1 -sulfonic acid (DSS) through the water resonance and processed as described [40]. Resonances of 1-D NMR spectra were fit to Lorentzian line shapes using the multipeak fitting package in Igor Pro 6.1.2.1.

\section{Resonance Raman spectroscopy}

Resonance Raman spectra were collected at room temperature with Soret excitation using a Coherent INNOVA 301C krypton ion laser at $406.7 \mathrm{~nm}$. The sample was placed on a Nikon Eclipse TE2000-U inverted microscope and the scattered light was collected at a $180^{\circ}$ geometry. The spectrum was dispersed with an Acton Research monochromator equipped with an 1800 blaze holographic diffraction grating and collected with a liquid nitrogen-cooled CCD camera (Princeton Instruments). The $200 \mu \mathrm{M}$ sample was placed in a quartz fluorescence cuvette with sample stirring to prevent localized heating. Laser power at the cell was between 15 and $25 \mathrm{~mW}$. Spectra were referenced linearly with solid sodium sulfate and resolution is within $1 \mathrm{~cm}^{-1}$. The spectra were fit to Lorentzian line shapes using a least-squares multipeak fitting procedure in Igor Pro 6.1.2.1 (Wavemetrics). A linear background was allowed to vary freely, along with the peak frequencies, intensities, and linewidths.

\section{EPR spectroscopy}

EPR spectroscopy was performed on a Brüker EMXplus EPR spectrometer equipped with a helium cryostat and temperature controller (Oxford Instruments). Protein samples were prepared in $50 \mathrm{mM}$ HEPES buffer at $\mathrm{pH}$ 7.0. Data were collected at $5 \mathrm{~K}$ with a microwave frequency of $9.382 \mathrm{GHz}$. Spectra were collected with a time constant of $163.84 \mathrm{~ms}$, a modulation frequency of $30 \mathrm{kHz}$, and a modulation amplitude of $6.090 \mathrm{G}$. Sweep times ranged from 100.26 to $401.1 \mathrm{~s}$. Data collected on $H t 1 \mathrm{X}$ averaged 16 scans with a microwave power of $100.2 \mu \mathrm{W}$ and a gain of $12 \mathrm{~dB}$. For $H t \mathrm{WT}$, eight scans were collected with a microwave power of $100.2 \mu \mathrm{W}$ and a gain of $15 \mathrm{~dB}$. Ht $3 \mathrm{X}$ required eight scans with a microwave power of $200 \mu \mathrm{W}$ and a gain of $35 \mathrm{~dB}$. Ht $4 \mathrm{X}$ 
required six scans with a microwave power of $50.24 \mu \mathrm{W}$ and a gain of $12 \mathrm{~dB}$. For each sample, the microwave power was tested to ensure the absence of saturation effects.

\section{Results and analysis}

\section{Effects of mutations on protein structure}

The perturbations to protein structure resulting from the heme attachment motif mutations were assessed using 2-D ${ }^{1} \mathrm{H}_{-}{ }^{15} \mathrm{~N}$ HSQC NMR spectra of uniformly ${ }^{15} \mathrm{~N}$-labeled protein samples. These spectra display a single cross peak for each backbone amide group, providing a fingerprint of the protein through which structural perturbations can be identified. The $\mathrm{Fe}^{\mathrm{II}}$ form of the protein was used to avoid the effects of changes in hyperfine (specifically pseudocontact) chemical shifts [41].

The HSQC spectra of the Ht $1 \mathrm{X}, \mathrm{Ht} 3 \mathrm{X}$, and $\mathrm{Ht} 4 \mathrm{X}$ variants are each overlayed with the spectrum of wild type (WT) in Figs. S1-S3, and the chemical shifts of assigned backbone amide ${ }^{1} \mathrm{H}$ and ${ }^{15} \mathrm{~N}$ nuclei are listed in Table $\mathrm{S} 1$. The spectra indicate that the mutants are well folded, but with some structural deviations from WT. The signal-to-noise in the $\mathrm{Ht}$ $1 \mathrm{X}$ spectrum is poor, because the low expression levels of this protein resulted in a low concentration of sample. The appearance of the HSQC spectrum of $H t 1 \mathrm{X}$ is suggestive of slow structural dynamics and/or conformational heterogeneity, as indicated by the doubling and/or broadening of some peaks. Nevertheless, the spectrum shows dispersion consistent with a well-folded protein. Assignments from the literature (BMRB Entry 10135) [42] assisted in the identification of amide backbone resonances with significant chemical shift deviations for each variant relative to wild type. The full sequence of each variant is shown in Fig. S4, where the amino acid letter designations in bold indicate backbone amide NMR chemical shifts that deviate significantly from those of $H t \mathrm{WT}$, or that are assigned in $H t \mathrm{WT}$ but not detected in the variant, suggesting a large shift in the chemical shift(s) and/or enhanced exchange with solvent. These effects are all consistent with the mutation exerting a significant effect on the environment of that amide. The changes are also highlighted pictorially for all three variants in Figs. S5-S7. We conclude that there are some structural perturbations as a result of the mutations, which is expected, but they are concentrated near the mutation site and do not significantly disrupt the overall protein fold.

The 1-D NMR spectra of the $\mathrm{Fe}^{\mathrm{III}}$ variants are shown in Fig. 4; small differences in hyperfine shifts are evident both in the high-frequency and low-frequency regions [33]. At low frequency, a prominent resonance for the axial Met61 ${ }_{\varepsilon}-\mathrm{C}^{1} \mathrm{H}_{3}$ is seen in all variants, confirming Met ligation to heme. The shifts and linewidths of the four heme methyl ${ }^{1} \mathrm{H}$

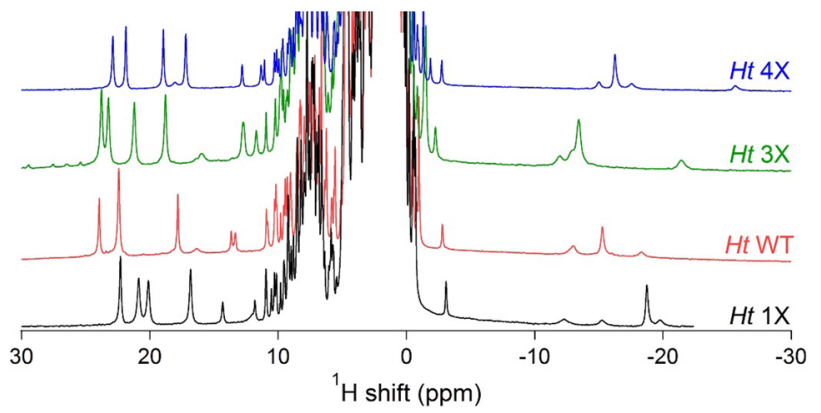

Fig. 4 1-D ${ }^{1} \mathrm{H}-\mathrm{NMR}$ spectra of the $\mathrm{Fe}^{\mathrm{III}} H t$ cyt $c_{552}$ heme attachment motif variants collected at $40{ }^{\circ} \mathrm{C}$

Table 1 Heme attachment motif sequences and reduction potentials of $H t$ cyt $c_{552}$ variants

\begin{tabular}{lll}
\hline Variant & Sequence & $\begin{array}{l}\text { Potential } \\
(\mathrm{mV} \text { vs. } \\
\text { SHE) }\end{array}$ \\
\hline $1 \mathrm{X}$ & $\mathrm{CGCH}$ & $130 \pm 6$ \\
$\mathrm{WT}$ & $\mathrm{CMACH}$ & $236 \pm 2$ \\
$3 \mathrm{X}$ & CMAGCH & $194 \pm 5$ \\
$4 \mathrm{X}$ & CAMAGCH & $75 \pm 6$ \\
\hline
\end{tabular}

peaks found at high frequency are consistent with His/Met axial ligation and a fluxional axial Met, sampling two conformations related by inversion through sulfur as previously described [43]. We conclude that His-Met axial ligation is maintained in the variants and is similar to that seen in WT.

\section{Reduction potential measurements}

The reduction potentials of the protein variants are presented in Table 1. The potential measured for $H t \mathrm{WT}, 236 \pm 2 \mathrm{mV}$, is consistent with the previous measurements $[19,38]$. The variants all have lower potentials than WT, with $H t 4 \mathrm{X}$ having the lowest of this group $(75 \mathrm{mV})$. The lowest potential previously reported for a well-folded variant of $H t$ cyt $c_{552}$ with axial His/Met ligands in place is $155 \mathrm{mV}$. This potential was measured for the double mutant $H t \mathrm{M} 13 \mathrm{~V} / \mathrm{K} 22 \mathrm{M}$ that was previously found to have enhanced heme ruffling relative to $H t$ WT [21, 40, 44]. This ruffling increase also has been proposed to contribute to the lower reduction potential in the mutant $[40,44]$.

\section{Heme and axial ligand chemical shift assignments}

Heme and axial ligand chemical shifts are assigned using the strategy described for $H t$ WT [40] and are reported in Table S2. To assess heme ruffling in the $H t 1 \mathrm{X}, 3 \mathrm{X}$, and $4 \mathrm{X}$ variants, the heme methyl, heme meso, and axial Met61 ${ }_{\varepsilon}-\mathrm{C}^{1} \mathrm{H}_{3}{ }^{1} \mathrm{H}$ shifts were measured for each variant in the $\mathrm{Fe}^{\mathrm{III}}$ 
$(S=1 / 2)$ state (see Fig. S8 for ligand atom nomenclature). In addition, the average values for the heme methyl, heme meso, heme $\alpha$-pyrrole, and heme $\beta$-pyrrole ${ }^{13} \mathrm{C}$ shifts were determined (see Fig. 3a for heme atom nomenclature). Assignment of the heme meso, $\alpha$-pyrrole, and $\beta$-pyrrole ${ }^{13} \mathrm{C}$ shifts made use of isotope labeling afforded by the biosynthetic incorporation of 5-aminolevulinic acid (ALA), as shown in Fig. 3. 1-D ${ }^{1} \mathrm{H}-\mathrm{NMR}$ spectra were used to measure the Met61 $\varepsilon-\mathrm{C}^{1} \mathrm{H}_{3}$ and the heme methyl ${ }^{1} \mathrm{H}$ shifts (Fig. 4). The heme methyl ${ }^{13} \mathrm{C}$ shifts were measured using ${ }^{1} \mathrm{H}-{ }^{13} \mathrm{C}$ HMQC spectra of a natural isotope abundance sample (Fig. S9) using the correlations between the HMQC peaks and the previously assigned ${ }^{1} \mathrm{H}$ resonances [45]. The heme meso ${ }^{1} \mathrm{H}$ and ${ }^{13} \mathrm{C}$ shifts were detected via ${ }^{1} \mathrm{H}_{-}{ }^{13} \mathrm{C}$ HMQC spectra of a sample with selected heme carbons including the meso carbons ${ }^{13} \mathrm{C}$-enriched by supplementation of the expression medium with $5-{ }^{13} \mathrm{C}$-ALA (Fig. S10). Four of the eight ${ }^{13} \mathrm{C} \beta$-pyrrole shifts and four of the eight ${ }^{13} \mathrm{C} \alpha$-pyrrole shifts were measured in $1-\mathrm{D}{ }^{13} \mathrm{C}-\mathrm{NMR}$ spectra of the $4-{ }^{13} \mathrm{C}$ ALA isotope-enriched samples (Fig. 5). The other four ${ }^{13} \mathrm{C}$ $\alpha$-pyrrole shifts were determined through inspection of 1-D ${ }^{13} \mathrm{C}-\mathrm{NMR}$ spectra of the $5-{ }^{13} \mathrm{C}$-ALA isotope-enriched sample (Fig. 6).

The chemical shifts of the axial histidine (His16) $\delta 1-{ }^{15} \mathrm{~N}$ and $\delta 1-\mathrm{N}^{1} \mathrm{H}$ (Fig. S8) were also assigned, identified by the peak with the largest ${ }^{1} \mathrm{H}$ and ${ }^{15} \mathrm{~N}$ chemical shifts observed in ${ }^{1} \mathrm{H}-{ }^{15} \mathrm{~N}$ HSQC spectra (Fig. S11) [44]. The His $16 \beta-{ }^{1} \mathrm{H}-{ }^{13} \mathrm{C}$ cross peaks were assigned by analogy to shifts of $H t \mathrm{WT}$ in natural isotope abundance HMQC spectra (Fig. S12), collected as described [40, 44]. All NMR data were collected at $40{ }^{\circ} \mathrm{C}$ to allow comparison to the existing data on other $\mathrm{Ht}$ cyt $c_{552}$ variants $[20,40]$ except for ${ }^{1} \mathrm{H}-{ }^{15} \mathrm{~N}$ HSQC spectra, which were collected at $25{ }^{\circ} \mathrm{C}$ to enhance the detection of ${ }^{1} \mathrm{H}-{ }^{15} \mathrm{~N}$ peaks by slowing exchange of the amide protons with solvent. The chemical shifts that are determined are shown in Table 2 where they are compared to those of $H t$ WT [20,

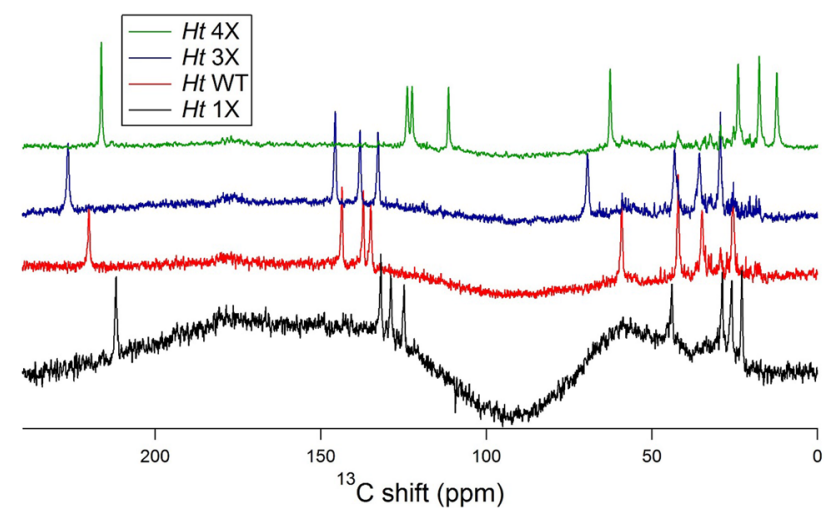

Fig. 5 1-D ${ }^{13} \mathrm{C}-\mathrm{NMR}$ spectra of the $\mathrm{Fe}{ }^{\mathrm{III}} H t$ cyt $c_{552}$ heme attachment motif variants collected at $40{ }^{\circ} \mathrm{C}$ with $4-{ }^{13} \mathrm{C}$-ALA isotope enrichment (Fig. 3a)

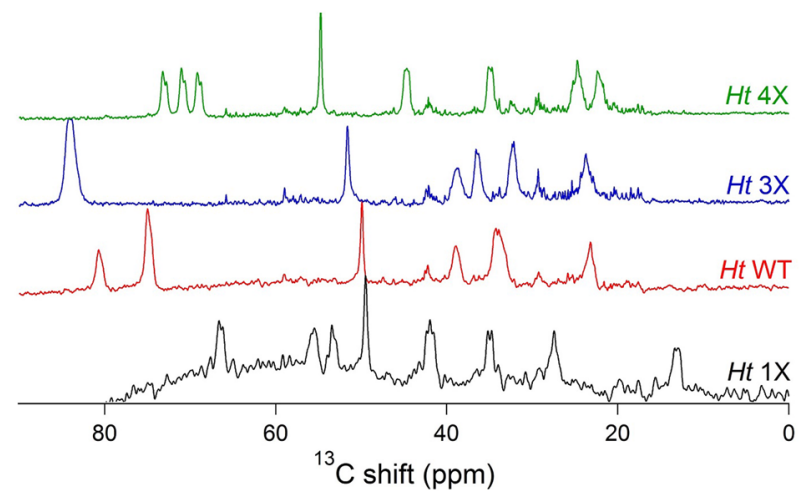

Fig. 6 1-D ${ }^{13} \mathrm{C}$-NMR spectra of the $\mathrm{Fe}{ }^{\mathrm{III}} H t$ cyt $c_{552}$ heme attachment motif variants collected at $40{ }^{\circ} \mathrm{C}$ with $5-{ }^{13} \mathrm{C}$-ALA isotope enrichment (Fig. 3b)

40]. Nucleus-specific assignments were not determined for all heme ${ }^{1} \mathrm{H}$ - and ${ }^{13} \mathrm{C}$-NMR shifts, and Table 2 reports the average chemical shifts for each type of heme nucleus. These averaged values were used in the analysis of heme ruffling as described previously [20, 40].

\section{NMR hyperfine shift analysis}

Prior studies have identified that heme hyperfine shifts depend on the presence and extent of heme ruffling when comparing cyts $c$ with His/Met axial ligation. These variations arise primarily from the modulation of the overlap between Fe $d(\pi)$ orbitals $\left(d_{x z}, y z\right)$ and porphyrin $e_{\mathrm{g}}$ orbitals,

Table 2 NMR chemical shifts (ppm) of $\mathrm{Fe}^{\mathrm{III}} H t$ cyt $c_{552}$ heme attachment motif variants

\begin{tabular}{lrrrr}
\hline & \multicolumn{1}{c}{$1 \mathrm{X}$} & \multicolumn{1}{c}{$\mathrm{WT}^{\mathrm{a}}$} & \multicolumn{1}{c}{$3 \mathrm{X}$} & \multicolumn{1}{c}{$4 \mathrm{X}$} \\
\hline Methyl ${ }^{1} \mathrm{H}$ & 20.0 & 21.6 & 21.7 & 20.2 \\
Methyl ${ }^{13} \mathrm{C}$ & -35.0 & -36.7 & -36.3 & -35.8 \\
Meso ${ }^{1} \mathrm{H}$ & 3.0 & 3.6 & 3.6 & 3.0 \\
Meso ${ }^{13} \mathrm{C}$ & 38.7 & 35.7 & 36.5 & 40.3 \\
$\alpha$-Pyrrole ${ }^{13} \mathrm{C}$ & 38.8 & 53.9 & 58.5 & 44.0 \\
$\beta$-Pyrrole ${ }^{13} \mathrm{C}^{\mathrm{b}}$ & 149.4 & 158.9 & 160.7 & 143.5 \\
Met61 $\varepsilon-\mathrm{C}^{1} \mathrm{H}_{3}$ & -19.0 & -15.3 & -13.4 & -16.3 \\
His16 $\delta_{1}{ }^{15} \mathrm{~N}^{\mathrm{c}}$ & 164.7 & 182.3 & 186.1 & 162.4 \\
His16 $\delta_{1}-\mathrm{N}^{1} \mathrm{H}^{\mathrm{c}}$ & 11.8 & 13.2 & 12.1 & 9.2 \\
His16 $\beta-{ }^{13} \mathrm{C}$ & 24.8 & 27.7 & 28.8 & 25.9 \\
\hline
\end{tabular}

Temperature is $40^{\circ} \mathrm{C}$ unless otherwise noted

For the heme nuclei, average shifts for nuclei of the type listed are reported

${ }^{\text {a } D a t a ~ f r o m ~ R e f . ~[40] ~}$

${ }^{\mathrm{b}}$ The average ${ }^{13} \mathrm{C}$ chemical shifts for four out of the eight $\beta$-pyrroles, which includes only those labeled via the $4-{ }^{13} \mathrm{C}$-ALA labeling scheme (Fig. 3)

${ }^{\mathrm{c}}$ Data collected at $25^{\circ} \mathrm{C}$ 
as well as the iron $d_{x y}$ orbital and the porphyrin $a_{1 \mathrm{u}}$ orbital $[20,40,46,47]$. These interactions impact the distribution of unpaired electron spin density on the heme and thus the observed hyperfine shifts, which are dominated by the Fermi contact term $[48,49]$. More specifically, as ruffling increases, the hyperfine shifts of the $\beta$-pyrrole carbons decrease, while spin densities on the $\alpha$-pyrrole carbons and meso carbons increase. In addition, ruffling decreases the hyperfine shifts of the heme methyl ${ }^{1} \mathrm{H}$ and ${ }^{13} \mathrm{C}$ nuclei, which receive spin density from the $\beta$-pyrrole carbons through spin polarization. The axial ligand shifts also show correlations with ruffling; these effects have been described in detail previously [20, 40,44]. An illustration of the relative changes in these chemical shifts with ruffling is provided in Fig. 7.

The changes in key chemical shifts of the three heme attachment motif variants of $H t$ cyt $c_{552}$ relative to $H t \mathrm{WT}$ are reported in Table 3. These shift changes are also compared to those observed in another variant of $H t$ cyt $c_{552}$, M13V/K22M [19], which was previously shown to have enhanced heme ruffling [21, 40, 44]. The use of average chemical shifts of nuclei of the same type (i.e., $\alpha$-pyrrole, $\beta$-pyrrole, and heme methyl) in this analysis corrects for the influence of asymmetric distribution of spin density on the heme resulting from heme-axial ligand interactions [50], making the average values a more useful indicator of ruffling than values of individual nuclei. The observed chemical shifts are used rather than the hyperfine shifts, which is the approach used in our prior work in which observed shifts were found to correlate well with ruffling [20, 40]. Using the observed shifts yields a convenient tool that can be used by non-experts to compare different heme protein variants. Furthermore, the diamagnetic contribution to these observed shifts is small and minimally impacted by mutations that alter heme conformation. It is also important to keep in mind that the analysis of heme ruffling performed

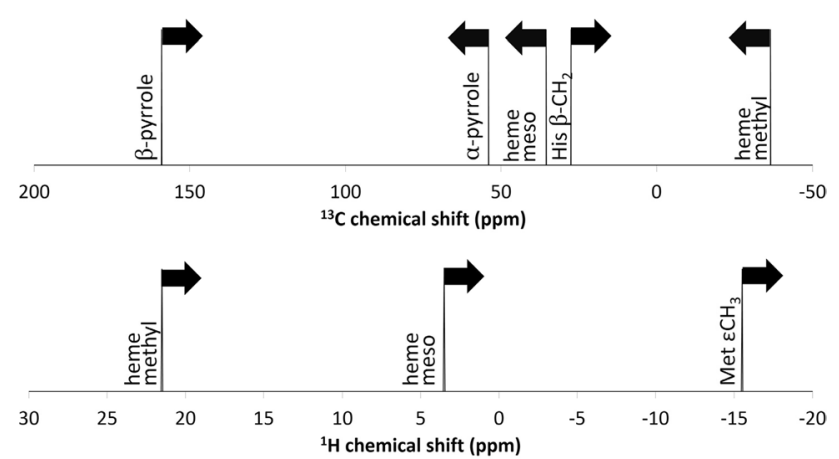

Fig. 7 Illustration of the relative changes of heme and axial ligand chemical shifts with increased ruffling. Values are shown for ${ }^{13} \mathrm{C}$ (top) and ${ }^{1} \mathrm{H}$ (bottom) nuclei of the heme and axial ligands. The placement on the chemical shift scales indicates a typical chemical shift for the nucleus or group indicated. The values indicated for heme nuclei are a typical average value for each type of nucleus
Table 3 NMR chemical shift changes (ppm) of $\mathrm{Fe}^{\mathrm{III}} H t$ cyt $c_{552}$ heme attachment motif variants relative to $H t$ WT

\begin{tabular}{lrrrr}
\hline & $\Delta(1 \mathrm{X})$ & $\Delta(3 \mathrm{X})$ & $\Delta(4 \mathrm{X})$ & $\Delta(\mathrm{M} 13 \mathrm{~V} / \mathrm{K} 22 \mathrm{M})^{\mathrm{a}}$ \\
\hline Methyl ${ }^{1} \mathrm{H}$ & -1.6 & +0.1 & -1.4 & -1.1 \\
Methyl ${ }^{13} \mathrm{C}$ & +1.7 & +0.4 & +0.9 & +0.2 \\
Meso ${ }^{1} \mathrm{H}$ & -0.6 & +0.0 & -0.7 & -0.5 \\
Meso ${ }^{13} \mathrm{C}$ & +3.0 & +0.8 & +4.6 & +1.1 \\
$\alpha$-Pyrrole ${ }^{13} \mathrm{C}$ & -15.1 & +4.7 & -9.9 & -12.0 \\
$\beta$-Pyrrole ${ }^{13} \mathrm{C}^{\mathrm{b}}$ & -9.5 & +1.8 & -15.4 & -12.2 \\
Met61 $\varepsilon-\mathrm{C}^{1} \mathrm{H}_{3}$ & -3.7 & +1.8 & -1.0 & -3.0 \\
His16 $\delta_{1^{-}}{ }^{15} \mathrm{~N}^{\mathrm{c}}$ & -17.6 & +3.8 & -19.9 & -15.2 \\
His16 $\delta_{1^{-}} \mathrm{N}^{1} \mathrm{H}^{\mathrm{c}}$ & -1.4 & -1.1 & -4.0 & -2.0 \\
His16 $\beta-{ }^{13} \mathrm{C}$ & -2.8 & +1.2 & -1.8 & -2.3 \\
\hline
\end{tabular}

Values are $\delta(\mathrm{WT})-\delta$ (mutant) determined using the chemical shifts reported in Table 2

For the heme nuclei, average shifts for nuclei of the type listed are reported

${ }^{\mathrm{a}} \mathrm{Ht} \mathrm{M} 13 \mathrm{~V} / \mathrm{K} 22 \mathrm{M}$ has previously been shown to have increased heme ruffling relative to $H t \mathrm{WT}$ [21]

${ }^{\mathrm{b}}$ The average ${ }^{13} \mathrm{C}$ chemical shifts for four out of the eight $\beta$-pyrroles, which includes only those labeled via the $4-{ }^{13} \mathrm{C}$-ALA labeling scheme (Fig. 3)

${ }^{\mathrm{c}}$ Data collected at $25^{\circ} \mathrm{C}$

here is qualitative and is based on the changes in hyperfine shifts that were observed previously in experiments and in DFT calculations [20,40]. Taken together, the changes in the heme chemical shifts indicate that both $H t 1 \mathrm{X}$ and $4 \mathrm{X}$ are more ruffled than $\mathrm{WT}$, while $H t 3 \mathrm{X}$ may be slightly less ruffled, but is similar to WT.

While the chemical shifts cannot be used to quantitatively deduce the amount of heme ruffling, the comparison of the relative shifts provides an indication of relative amounts of heme ruffling among a series of protein variants. The average heme methyl ${ }^{1} \mathrm{H}$ shift has been found to be a particularly reliable indicator of the relative amount of heme ruffling [20, 40]. Based on the magnitudes of the changes in the average heme methyl ${ }^{1} \mathrm{H}$ shift, the increase in ruffling of $\mathrm{Ht} 1 \mathrm{X}$ and $H t 4 \mathrm{X}$ relative to $H t \mathrm{WT}$ is greater than that observed for $H t$ $\mathrm{M} 13 \mathrm{~V} / \mathrm{K} 22 \mathrm{M}$, the $H t$ cyt $c_{552}$ mutant that previously was found to have the largest amount of ruffling and the lowest reduction potential among mutants of this protein with His/Met axial ligation [21]. This conclusion is corroborated by the changes in the average ${ }^{13} \mathrm{C}$ heme methyl and heme mesoshifts, which also have been found to be reasonable predictors of heme ruffling $[20,40]$. While considering these results, it is important to keep in mind that structural changes other than changes in heme ruffling will be induced by mutations, and any such changes also may impact reduction potential.

$\mathrm{X}$-ray crystallography is a valuable complementary method for characterizing heme conformation and can 
yield quantitative results. A major advantage of X-ray crystallography is that it reveals all types of heme distortions (i.e., saddling, doming, and other distortions in addition to ruffling). In our analysis here, it is possible that distortions other than ruffling are present but not detected. However, there are disadvantages to the use of crystallography to measure heme distortion. The most significant issue is that $\mathrm{X}$-ray crystal structures of proteins rarely have the high resolution needed for a robust assessment of heme conformation. Small changes in heme distortion may not be discernable in a crystal structure but will still impact hyperfine shifts. Ultimately, combining multiple methods is expected to give the best results.

\section{Resonance Raman spectroscopy}

Resonance Raman (rR) spectra collected for ferric $H t$ WT and the heme attachment variants $H t 1 \mathrm{X}, 3 \mathrm{X}$, and $4 \mathrm{X}$ are shown in Fig. 8. In addition, the rR spectra of the variants $H t \mathrm{M} 13 \mathrm{~V}, \mathrm{~K} 22 \mathrm{M}$, and M13V/K22M [19] are measured (Fig. $\mathrm{S} 13)$. These variants are shown previously to have increased heme ruffling relative to $H t \mathrm{WT}$, and the double mutant has the greatest ruffling [21]. An example of the results of peak fitting to determine band positions (Table 4) is shown in Fig. S14. The high-frequency resonance Raman marker bands have been assigned previously [51]. Here, we provide the energies of two bands, $\nu_{4}$ and $\nu_{10}$. The $\nu_{4}$ band is sensitive to heme oxidation state and is provided to show that samples remain oxidized in this experiment. Of particular interest for the analysis of ruffling is the $\nu_{10}$ frequency, which is sensitive to the porphyrin ruffling distortion [27, 52]. In the mutants proposed to have greater ruffling than WT, the $\nu_{10}$ band shifts to slightly lower energy. A similar shift, however,

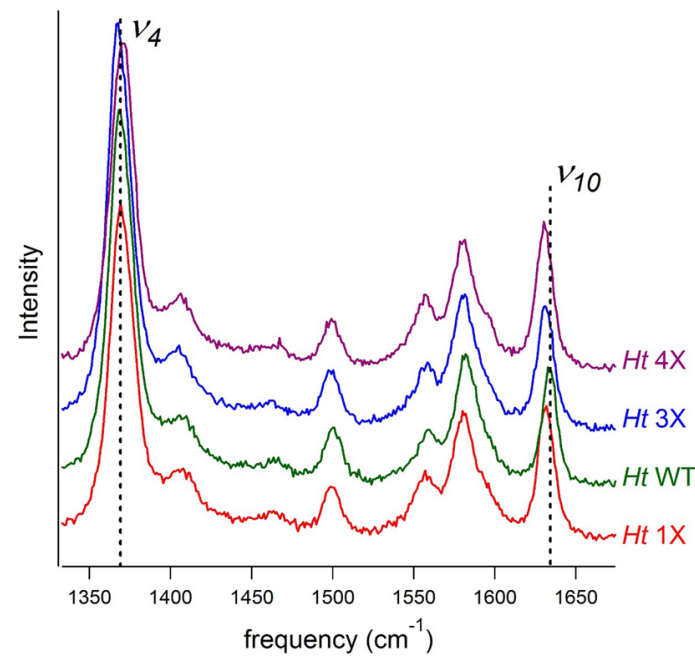

Fig. 8 High-frequency region of the resonance Raman spectra for the $\mathrm{Fe}^{\mathrm{III}} H t$ cyt $c_{552}$ heme attachment motif variants
Table 4 Resonance Raman $\nu_{4}$ and $\nu_{10}$ peak frequencies for $\mathrm{Fe}^{\mathrm{III}} \mathrm{Ht}$ cyt $c_{552}$ variants

\begin{tabular}{lll}
\hline$H t$ variant & $\nu_{4}\left(\mathrm{~cm}^{-1}\right)$ & $\nu_{10}\left(\mathrm{~cm}^{-1}\right)$ \\
\hline $1 X$ & 1370 & 1631 \\
WT & 1369 & 1634 \\
$3 X$ & 1368 & 1631 \\
$4 X$ & 1370 & 1630 \\
K22M & 1370 & 1634 \\
M13V & 1370 & 1633 \\
M13V/K22M & 1370 & 1633 \\
\hline
\end{tabular}

is also seen for $H t 3 \mathrm{X}$, which NMR data which suggest has a heme conformation that is similar to WT, or slightly more planar. Overall, the changes in the rR spectra of these variants are small, and we conclude that $\mathrm{rR}$ is significantly less sensitive to heme ruffling for these systems than NMR. Nevertheless, $\mathrm{rR}$ has been successfully applied to other systems to assess ruffling. In particular, rR spectra are quite sensitive to changes in ruffling for porphyrins that are already highly ruffled [53]. The systems studied here have a modest amount of ruffling ( $0.62 \AA$ out-of-plane distortion along the ruffling coordinate for $H t$ WT [20]; in this range, rR frequencies) change minimally with alterations in ruffling.

\section{EPR spectroscopy}

The EPR spectra of the $H t$ cyt $c_{552}$ heme attachment motif variants are shown in Fig. 9. The spectra are consistent with low-spin, $\mathrm{Fe}^{\mathrm{III}}$ species. The $g_{\max }$ and $g_{\text {mid }}$ values were fit to

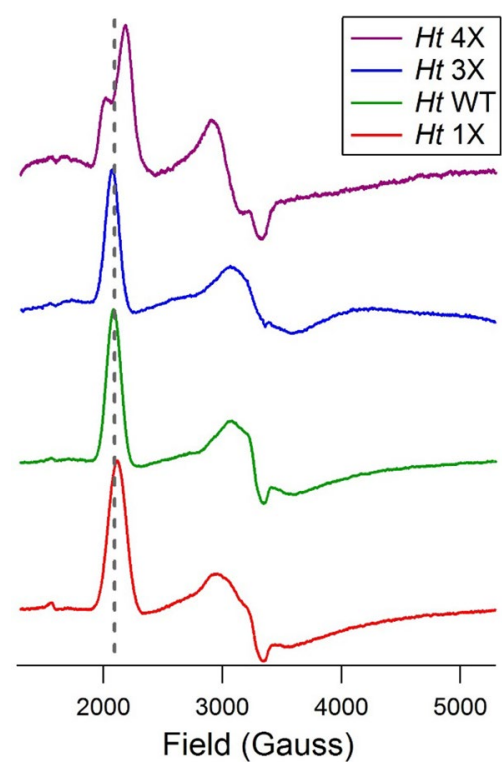

Fig. $9 \mathrm{X}$-band EPR spectra of Fe $\mathrm{III}^{\mathrm{III}} H t$ cyt $c_{552}$ heme attachment variants 
the EPR spectra using SimPow6. The $g_{\min }$ values are not well resolved, which is attributed to $g$-strain, and are calculated using the following normalization condition that applies to a $\left(d_{x y}\right)^{2}\left(d_{x z, y z}\right)^{3}$ ground state $[54,55]$ :

$g_{x}^{2}+g_{y}^{2}+g_{z}^{2}+g_{y} g_{z}-g_{x} g_{z}-g_{x} g_{y}-4\left(g_{x}+g_{y}-g_{z}\right)=0$.

The $g$ values determined here are given in Table 5, where they are compared with the $g$ values of other $H t$ cyt $c_{552}$ variants determined previously to have altered heme ruffling [21]. The $g_{\max }$ value, which is sensitive to the extent of heme ruffling [21, 56-58], shows a significant deviation among the variants. In the absence of influences from changes in axial ligand orientation, heme ruffling is expected to increase the rhombicity of the EPR signal, decreasing $g_{\max }$, which is the relationship observed for $H t 1 \mathrm{X}$ and $H t 4 \mathrm{X}$ (Table 5). In contrast, $H t 3 \mathrm{X}$ has a slightly higher $g_{\max }$ than $H t \mathrm{WT}$. These findings are broadly consistent with the conclusions drawn from NMR data; namely, $H t 1 \mathrm{X}$ and $4 \mathrm{X}$ have increased ruffling; Ht $3 \mathrm{X}$ has slightly decreased or unchanged ruffling. For Ht 4X, we note that there are two components of the EPR signal, indicating sample heterogeneity and/or chemical exchange.

\section{Discussion}

\section{Spectroscopic probes of heme ruffling}

Resonance Raman (rR) has been considered a key spectroscopic probe of heme conformation, with the $\nu_{10}$ energy used as an indicator of ruffling [27, 52]. Here, we utilize rR along with NMR and EPR of paramagnetic $(S=1 / 2)$ cyts $c$ to investigate heme conformation changes induced by

Table 5 EPR-determined $g$ values of the $H t$ cyt $c_{552}$ variants

\begin{tabular}{llll}
\hline$H t$ variant & $g_{\max }$ & $g_{\text {mid }}$ & $g_{\text {min }}^{\mathrm{a}}$ \\
\hline $1 \mathrm{X}$ & 3.17 & 2.11 & 1.23 \\
$\mathrm{WT}^{\mathrm{b}}$ & 3.21 & 2.07 & 1.17 \\
$3 \mathrm{X}$ & 3.23 & 2.06 & 1.13 \\
$4 \mathrm{X}$ (major component) & $3.06^{\mathrm{c}}$ & - & - \\
$4 \mathrm{X}$ (minor component) & $3.28^{\mathrm{c}}$ & - & - \\
$\mathrm{K}_{2} 2 \mathrm{M}^{\mathrm{d}}$ & 3.23 & 2.08 & 1.19 \\
$\mathrm{M} 13 \mathrm{~V}^{\mathrm{d}}$ & 3.19 & 2.09 & 1.17 \\
$\mathrm{M} 13 \mathrm{~V} / \mathrm{K} 22 \mathrm{M}^{\mathrm{d}}$ & 3.17 & 2.11 & 1.17 \\
\hline
\end{tabular}

${ }^{\mathrm{a} C a l c u l a t e d}$ from Eq. 1

${ }^{\mathrm{b}}$ Also reported in Refs. [20, 21]

${ }^{\mathrm{c}}$ The presence of two components prevented a reliable fit of the $g$ values other than $g_{\max }$

${ }^{\mathrm{d}}$ Reported in Ref. [21] mutations to the heme attachment motif. NMR hyperfine shifts are particularly sensitive to these changes. These shifts result from unpaired electron spin delocalization onto the porphyrin and axial ligands (directly or through spin polarization) and from a through-space dipolar electron-nucleus interaction [59-61]. Heme ruffling has been shown to modulate both of these factors, resulting in a largely predictable pattern of hyperfine shift changes with ruffling [20, 40]. Fortuitously, the average heme methyl ${ }^{1} \mathrm{H}$ shift (which is the most convenient shift to measure) has been found to be the most reliable predictor of ruffling in ferricyts $c$, with this shift decreasing as ruffling increases [40]. The $g_{\max }$ value also has been found to track well with the changes in hyperfine shifts and with heme ruffling, provided that axial ligand type and orientation are conserved. In particular, more ruffled cyts $c$ are more rhombic and have a lower $g_{\max }$ value [21, 56-58]. For comparison purposes, we also collected rR spectra. While the changes in the $\nu_{10}$ marker band are broadly consistent with the changes in the NMR and EPR data, with a lower energy observed for more ruffled protein variants, the changes in this band are on the order of only a few $\mathrm{cm}^{-1}$, while NMR and EPR spectra show significantly greater changes. We also note that UV-Vis absorption data also have been implemented as a probe of ruffling, but large changes in ruffling are required to see any shift in absorption bands [62].

Of the protein variants studied here, $H t 1 \mathrm{X}$ has the largest decrease in average heme methyl ${ }^{1} \mathrm{H}$ shift relative to wild type, suggesting that it has the largest increase in ruffling. The $g_{\max }$ value of $H t 1 \mathrm{X}$ is decreased, as well, which is consistent with this conclusion. In addition, the $H t 1 \mathrm{X} \mathrm{rR} \nu_{10}$ band shifts to slightly lower energy, which is also consistent with more ruffling. We propose that this variant shows a higher amount of ruffling due to strain introduced in the CGCH linker.

$\mathrm{Ht} 4 \mathrm{X}$ displays the second greatest change in ruffling, at least as indicated by its decrease in average ${ }^{1} \mathrm{H}$ methyl shift compared to WT. Interestingly, the EPR spectrum of $\mathrm{Ht} 4 \mathrm{X}$ shows two components: one with a high $g_{\max }(3.28)$ and one with a low $g_{\max }$ (3.06). This heterogeneity is not observed by NMR at $40{ }^{\circ} \mathrm{C}$, which suggests that it is a result of fast chemical exchange on the NMR time scale at $40{ }^{\circ} \mathrm{C}$ between two states that are frozen out at the low temperature at which the EPR spectrum was measured. The major component has the lower $g_{\max }$, which is similar to the value for the highly ruffled horse cytochrome $c(1.0 \AA$ out-of plane ruffling distortion) $[58,63]$. The minor component has a $g_{\max }$ value of 3.28 , which is close to the range observed for highly axial cyts $c$ and is attributed at least in part to a relatively planar heme [56-58]. Finally, the $\mathrm{rR} \nu_{10}$ band also shifts to lower energy in $H t 4 \mathrm{X}$ and is the lowest among this series of variants, although the shift is very small (Table 4). We conclude that the heme of $H t 4 \mathrm{X}$ is in exchange between ruffled and 
planar conformations, but with a higher population of the ruffled form. While these forms are frozen out at the low temperature at which EPR spectra are collected, they are in fast exchange on the NMR and rR time scales near room temperature. A similar dynamic heme ruffling phenomenon has been observed in other heme proteins $[64,65]$ and metalloporphyrins [66]. Thus, we propose that extending the heme attachment motif adds strain to this system to enhance heme ruffling and also introduces heme conformational heterogeneity or dynamics. It is worth noting that the introduction of such dynamics typically complicates structural characterization using X-ray crystallography. The crystallization of proteins with dynamic heterogeneity may be challenging, and if crystals are obtained, there is a concern that one conformer may be crystallized preferentially. For these reasons, spectroscopic analysis of heme conformation has distinct advantages for dynamic systems like $H t 4 \mathrm{X}$.

Using the previously defined trends in hyperfine NMR shifts, the Ht $3 \mathrm{X}$ variant displays a similar degree of ruffling as $H t \mathrm{WT}$, or it may be slightly less ruffled. This is consistent with the similar $g_{\max }$ value of $H t \mathrm{WT}$ and $H t 3 \mathrm{X}$ (Table 5). The resonance Raman $\nu_{10}$ frequency of $H t 3 \mathrm{X}$ decreases slightly, which would be indicative of an increase in ruffling, but the average heme methyl chemical shift increases by $0.1 \mathrm{ppm}$, which suggests a slightly more planar heme. The data are broadly consistent with a similar degree of heme ruffling between $\mathrm{Ht} 3 \mathrm{X}$ and WT.

\section{Heme ruffling and reduction potential}

Heme ruffling has been proposed to lower reduction potential $[20,67,68]$, and a general correlation between heme ruffling and potential in cyts $c$ has been observed [15]. The multitude of factors that influence potential, including heme solvent exposure and local electrostatics, makes it difficult to correlate any single factor to determining the potential. To minimize the number of factors that can influence potential, we have investigated series of cyt $c$ variants in which specific mutations are introduced to influence the extent of heme ruffling. Earlier work utilized mutations near the CXXCH motif $[19,20]$. Here, we investigate the influence of the motif itself.

Each heme attachment motif variant of $H t$ cyt $c_{552}$ studied here has a decreased heme reduction potential. However, the $H t 1 \mathrm{X}$ and $4 \mathrm{X}$ variants, which were found to have a substantial increase in heme ruffling, also have larger decreases in potential (Table 1). In fact, a general correlation is observed between the average ${ }^{1} \mathrm{H}$ heme methyl shift, which is sensitive to heme ruffling, and the reduction potential of these and other $H t$ cyt $c_{552}$ variants (Fig. 10). A total range of $171 \mathrm{mV}$ is observed in the potentials of these $H t$ cyt $c_{552}$ variants [20]. In these mutants, major changes in heme solvent exposure are not expected, because the ${ }^{1} \mathrm{H}-{ }^{15} \mathrm{~N}$ HSQC

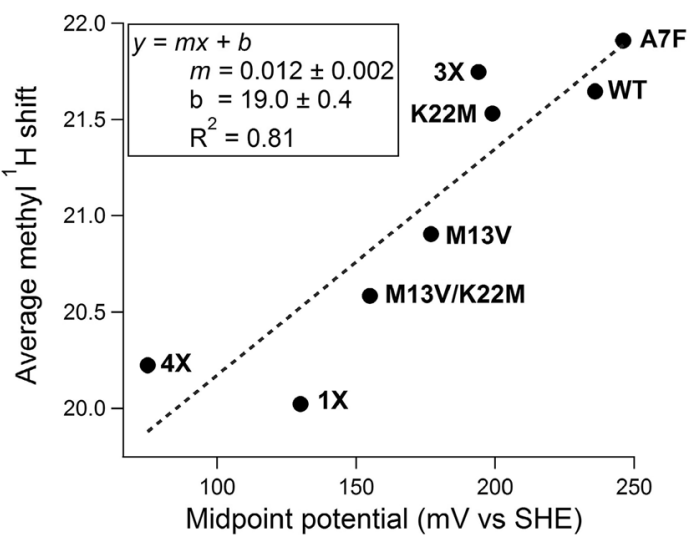

Fig. 10 Correlation between average ${ }^{1} \mathrm{H}$ heme methyl shift and the reduction potential of $H t$ cyt $c_{552}$ and the $1 \mathrm{X}, 3 \mathrm{X}, 4 \mathrm{X}, \mathrm{M} 13 \mathrm{~V}, \mathrm{~K} 22 \mathrm{M}$, $\mathrm{M} 13 \mathrm{~V} / \mathrm{K} 22 \mathrm{M}$, and A7F variants. Potentials of M13V, K22M, and $\mathrm{M} 13 \mathrm{~V} / \mathrm{K} 22 \mathrm{M}$ were reported elsewhere [44], and the potential of A7F was measured by PFV to be $246 \mathrm{mV}$. Chemical shifts were measured at $40{ }^{\circ} \mathrm{C}$

spectra reveal that the mutants do not have a significantly perturbed fold (Figs. S1-S7); however, changes in heme solvent interactions cannot be ruled out, in particular for $\mathrm{Ht}$ $1 X$. Significant changes in electrostatic interactions are only expected for the variants involving K22, as the other mutations introduced in this series are charge-neutral. Therefore, other factors determining potential are expected to yield the differences observed among these $H t$ cyt $c_{552}$ variants. The broad correlation between heme ruffling and reduction potential supports the hypothesis that heme ruffling plays a role in tuning the reduction potential. An explanation for this relationship has been put forward previously. Specifically, it was found through DFT calculations validated by NMR that enhancing ruffling destabilizes the $d_{\pi}$ orbitals of iron, but the net destabilization is greater for ferrous heme compared to ferric heme, thus yielding a lower reduction potential [20].

\section{Functional implications}

Extended heme attachment motifs in the form of $\mathrm{CX}_{3} \mathrm{CH}$ or $\mathrm{CX}_{4} \mathrm{CH}$ occur in tetraheme cyts $c_{3}$ of sulfate-reducing bacteria [69] and in a few other cyts $c$ [70]. In an analysis of heme conformation performed on cyts $c_{3}$, it was discovered that hemes 2 and 4 , which have extended heme attachment motifs, tend to be significantly more ruffled than hemes 1 and hemes 3 [69]. In this work, engineering an extended $\mathrm{CX}_{4} \mathrm{CH}$ heme attachment motif in $\mathrm{Ht}$ cyt $c_{552}$ increases heme ruffling and decreases potential. When including additional data from the literature on variants of $H t$ cyt $c_{552}$, a general correlation is observed between the average heme methyl shift, which tracks with heme ruffling, and reduction potential (Fig. 10). These findings suggest that naturally occurring extended heme attachment 
motifs in multiheme cyts $c$ may exist to tune the potential of the heme downward by altering the heme conformation. Indeed, it has been noted that $c$-type heme sites in nature span a wider range of reduction potentials relative to $b$-type hemes, comparing hemes with the same axial ligands. In particular, hemes $c$ reach lower potentials compared to hemes $b$ [15].

EPR data reveal that the heme of $H t 4 \mathrm{X}$ is in exchange between ruffled and planar conformations, a phenomenon that has been observed in some heme proteins $[64,65]$ and metalloporphyrins [66]. This kind of heme conformational dynamics has been subscribed functional significance in a heme-degrading enzyme [65]. In addition, interaction of mitochondrial cyt $c$ with membranes has been invoked in driving a conformational change of heme from ruffled to more planar. In particular, a decrease in heme ruffling has been observed in mitochondrial cyt $c$ bound to the mitochondrial membrane [71], and these changes have been proposed to enhance electronic coupling to redox partners [23]. Furthermore, heme conformational dynamics have been shown to couple strongly to the cyt $c$ polypeptide, and in particular to the protein region that interacts with redox partners [22, 24]. Thus, modulation of a ruffled/planar equilibrium may act as a "switch" for electron transfer [15]. While the functional relevance in cyt $c_{3}$ is not apparent, future studies of electron transfer in cyt $c_{3}$ should consider these possible effects on the hemes with the extended attachment motif.

\section{Implications for engineering heme peptides}

Heme peptides, or microperoxidases, consist of heme $c$ bound to a short peptide via a CXXCH attachment motif. Microperoxidases have long been a favorite model of heme protein active sites [72]. In addition, they have been employed in biotechnological applications, including hydrogen peroxide sensing [73-75] and biofuel cells [76-78]. In addition, cobalt substitution of a microperoxidase-11, derived from horse cyt $c$, yields an air-tolerant artificial hydrogenase for generating hydrogen from neutral $\mathrm{pH}$ water [36]. For applications involving immobilized metalloporphyrin peptides, the small size of heme peptides allows for a high density of active sites and resistance to deactivation due to irreversible unfolding, because the short peptides do not fold. However, tuning the properties of these metalloporphyrin-peptide catalysts has been a challenge because of the limited number of second-sphere interactions that exist and can be introduced. The ability to modulate the porphyrin conformation and, therefore, the reduction potential of microperoxidases by expression of microperoxidases with contracted or extended heme attachment motifs may allow the introduction of novel and/or altered activities of these mini-biomolecules [35].

\section{Summary and conclusions}

Extended $\left(\mathrm{CX}_{3} \mathrm{CH}\right.$ and $\left.\mathrm{CX}_{4} \mathrm{CH}\right)$ and contracted $(\mathrm{CXCH})$ heme attachment motifs in $H t$ cyt $c_{552}$ were evaluated in terms of their heme conformation using hyperfine NMR shifts, EPR $g_{\max }$ values, and resonance Raman $\nu_{10}$ frequencies. The heme in the $\mathrm{CX}_{4} \mathrm{CH}$ variant demonstrates enhanced ruffling, mimicking the effect observed in hemes in cyts $c_{3}$ with extended heme attachment motifs. The reduction potentials of the variants were also analyzed, showing a general correlation between higher heme ruffling and lower redox potential, adding an additional support for the hypothesis that ruffling lowers potential. Tuning redox potential through modification of the heme attachment motif length, which changes heme ruffling, may be a functional role of the extended heme attachment motifs in cytochromes $c_{3}$. The $\mathrm{CXCH}$ motif variant also notably increases the heme ruffling and lowers the redox potential. Modifying heme attachment motifs may also be an approach for tuning the properties of metalloporphyrin-peptide catalysts by altering the heme conformation.

Acknowledgements This work is supported by the Chemical Sciences, Geosciences and Biosciences Division, Office of Basic Energy Sciences, Office of Science, U.S. Department of Energy, Grant No. DE-FG02-09ER16121.

\section{References}

1. Bowman SEJ, Bren KL (2008) Nat Prod Rep 25:1118-1130

2. Einsle O, Messerschmidt A, Stach P, Bourenkov GP, Bartunik HD, Huber R, Kroneck PMH (1999) Nature 400:476-480

3. Arciero DM, Hooper AB (1994) J Biol Chem 269:11878-11886

4. Shimizu H, Schuller DJ, Lanzilotta WN, Sundaramoorthy M, Arciero DM, Hooper AB, Poulos TL (2001) Biochemistry 40:13483-13490

5. Wang YT, Graichen ME, Liu AM, Pearson AR, Wilmot CM, Davidson VL (2003) Biochemistry 42:7318-7325

6. Kagan VE, Tyurin VA, Jiang JF, Tyurina YY, Ritov VB, Amoscato AA, Osipov AN, Belikova NA, Kapralov AA, Kini V, Vlasova II, Zhao Q, Zou MM, Di P, Svistunenko DA, Kurnikov IV, Borisenko GG (2005) Nat Chem Biol 1:223-232

7. Allen JWA (2011) FEBS J 278:4198-4216

8. Simon J, Hederstedt L (2011) FEBS J 278:4179-4188

9. Stevens JM, Mavridou DAI, Hamer R, Kritsiligkou P, Goddard AD, Ferguson SJ (2011) FEBS J 278:4170-4178

10. Babbitt SE, Sutherland MC, Francisco BS, Mendez DL, Kranz RG (2015) Trends Biochem Sci 40:446-455

11. Gabilly ST, Hamel PP (2017) Front Plant Sci 8:1313

12. Allen JWA, Barker PD, Daltrop O, Stevens JM, Tomlinson EJ, Sinha N, Sambongi Y, Ferguson SJ (2005) Dalton Trans. https ://doi.org/10.1039/b508139b (ISSN 1477-9226:3410-3418)

13. Asher WB, Bren KL (2012) Chem Commun 48:8344-8346

14. Mavridou DAI, Ferguson SJ, Stevens JM (2013) IUBMB Life 65:209-216

15. Kleingardner JG, Bren KL (2015) Acc Chem Res 48:1845-1852 
16. Jentzen W, Song XZ, Shelnutt JA (1997) J Phys Chem B 101:1684-1699

17. Ma JG, Laberge M, Song XZ, Jentzen W, Jia SL, Zhang J, Vanderkooi JM, Shelnutt JA (1998) Biochemistry 37:5118-5128

18. Shokhireva TK, Berry RE, Uno E, Balfour CA, Zhang HJ, Walker FA (2003) Proc Natl Acad Sci USA 100:3778-3783

19. Michel LV, Ye T, Bowman SEJ, Levin BD, Hahn MA, Russell BS, Elliott SJ, Bren KL (2007) Biochemistry 46:11753-11760

20. Liptak MD, Wen X, Bren KL (2010) J Am Chem Soc 132:9753-9763

21. Can M, Zoppellaro G, Andersson KK, Bren KL (2011) Inorg Chem 50:12018-12024

22. Galinato MGI, Kleingardner JG, Bowman SEJ, Alp EE, Zhao J, Bren KL, Lehnert N (2012) Proc Natl Acad Sci USA 109:8896-8900

23. Sun Y, Benabbas A, Zeng W, Kleingardner JG, Bren KL, Champion PM (2014) Proc Natl Acad Sci USA 111:6570-6575

24. Galinato MGI, Bowman SEJ, Kleingardner JG, Martin S, Zhao J, Sturhahn W, Alp EE, Bren KL, Lehnert N (2015) Biochemistry 54:1064-1076

25. Bren KL (2016) Isr J Chem 56:693-704

26. Hobbs JD, Shelnutt JA (1995) J Protein Chem 14:19-25

27. Ma JG, Zhang J, Franco R, Jia SL, Moura I, Moura JJG, Kroneck PMH, Shelnutt JA (1998) Biochemistry 37:12431-12442

28. Travaglini-Allocatelli C, Gianni S, Dubey VK, Borgia A, Di Matteo A, Bonivento D, Cutruzzolà F, Bren KL, Brunori M (2005) J Biol Chem 280:25729-25734

29. Cheng RJ, Chen PY, Gau PR, Chen CC, Peng SM (1997) J Am Chem Soc 119:2563-2569

30. Shelnutt JA, Song XZ, Ma JG, Jia SL, Jentzen W, Medforth CJ (1998) Chem Soc Rev 27:31-41

31. Jentzen W, Ma JG, Shelnutt JA (1998) Biophys J 74:753-763

32. Hasegawa J, Yoshida T, Yamazaki T, Sambongi Y, Yu Y, Igarashi Y, Kodama T, Yamazaki K, Kyogoku Y, Kobayashi Y (1998) Biochemistry 37:9641-9649

33. Kleingardner JG, Bren KL (2011) Metallomics 3:396-403

34. Braun M, Thöny-Meyer L (2004) Proc Natl Acad Sci USA 101:12830-12835

35. Kleingardner EC, Asher WB, Bren KL (2017) Biochemistry 56:143-148

36. Kleingardner JG, Kandemir B, Bren KL (2014) J Am Chem Soc 136:4-7

37. Rivera M, Walker FA (1995) Anal Biochem 230:295-302

38. Ye T, Kaur R, Wen X, Bren KL, Elliott SJ (2005) Inorg Chem 44:8999-9006

39. Fourmond V, Hoke K, Heering HA, Baffert C, Leroux F, Bertrand P, Leger C (2009) Bioelectrochemistry 76:141-147

40. Kleingardner JG, Bowman SEJ, Bren KL (2013) Inorg Chem 52:12933-12946

41. Bren KL (2007) In: Scott RA, Lukehart CM (eds) Application of physical methods to inorganic and bioinorganic chemistry. Wiley, Chichester, pp 357-384

42. Takayama SJ, Takahashi Y, Mikami S, Irie K, Kawano S, Yamamoto Y, Hemmi H, Kitahara R, Yokoyama S, Akasaka K (2007) Biochemistry 46:9215-9224

43. Zhong L, Wen X, Rabinowitz TM, Russell BS, Karan EF, Bren KL (2004) Proc Natl Acad Sci USA 101:8637-8642

44. Bowman SEJ, Bren KL (2010) Inorg Chem 49:7890-7897

45. Karan EF, Russell BS, Bren KL (2002) J Biol Inorg Chem 7:260-272

46. Nakamura M (2006) Coord Chem Rev 250:2271-2294

47. Shokhireva TK, Shokhirev NV, Berry RE, Zhang HJ, Walker FA (2008) J Biol Inorg Chem 13:941-959
48. Walker FA (2003) Inorg Chem 42:4526-4544

49. Bren KL (2015) In: Swart M, Costas M (eds) Spin states in biochemistry and inorganic chemistry: influence on structure and reactivity. Wiley, Chichester, pp 409-434

50. Shokhirev NV, Walker FA (1998) J Biol Inorg Chem 3:581-594

51. Hu SZ, Morris IK, Singh JP, Smith KM, Spiro TG (1993) J Am Chem Soc 115:12446-12458

52. Czernuszewicz RS, Li XY, Spiro TG (1989) J Am Chem Soc 111:7024-7031

53. Song XZ, Jentzen W, Jia SL, Jaquinod L, Nurco DJ, Medforth CJ, Smith KM, Shelnutt JA (1996) J Am Chem Soc 118:12975-12988

54. Taylor CPS (1977) Biochim Biophys Acta 491:137-149

55. Castner TJ Jr (1959) Phys Rev 115:1506-1515

56. Zoppellaro G, Harbitz E, Kaur R, Ensign AA, Bren KL, Andersson KK (2008) J Am Chem Soc 130:15348-15360

57. Zoppellaro G, Bren KL, Ensign AA, Harbitz E, Kaur R, Hersleth H-P, Ryde U, Hederstedt L, Andersson KK (2009) Biopolymers 91:1064-1082

58. Can M, Krucinska J, Zoppellaro G, Andersen NH, Wedekind JE, Hersleth H-P, Andersson KK, Bren KL (2013) ChemBioChem 14:1828-1838

59. La Mar GN, Horrocks WD Jr, Holm RH (eds) (1973) NMR of paramagnetic molecules: principles and applications. Academic, New York

60. Bertini I, Luchinat C (1986) NMR of paramagnetic molecules in biological systems. Benjamin Cummings, Menlo Park

61. Walker FA (1999) Coord Chem Rev 186:471-534

62. Graves AB, Graves MT, Liptak MD (2016) J Phys Chem B 120:3844-3853

63. Brautigan DL, Feinberg BA, Hoffman BM, Margoliash E, Peisach J, Blumberg WE (1977) J Biol Chem 252:574-582

64. Yang F, Knipp M, Shokhireva TK, Berry RE, Zhang HJ, Walker FA (2009) J Biol Inorg Chem 14:1077-1095

65. Graves AB, Horak EH, Liptak MD (2016) Dalton Trans 45:10058-10067

66. Anderson KK, Hobbs JD, Luo LA, Stanley KD, Quirke JME, Shelnutt JA (1993) J Am Chem Soc 115:12346-12352

67. Barkigia KM, Chantranupong L, Smith KM, Fajer J (1988) J Am Chem Soc 110:7566-7567

68. Maes EM, Roberts SA, Weichsel A, Montfort WR (2005) Biochemistry 44:12690-12699

69. Aragao D, Frazao C, Sieker L, Sheldrick GM, LeGall J, Carrondo MA (2003) Acta Crystallogr Sect D Biol Crystallogr 59:644-653

70. Devreese B, Brige A, Backers K, Van Driessche G, Meyer TE, Cusanovich MA, Van Beeumen JJ (2000) Arch Biochem Biophys 381:53-60

71. Berezhna S, Wohlrab H, Champion PM (2003) Biochemistry 42:6149-6158

72. Marques HM (2007) Dalton Trans 39:4371-4385

73. Gong C, Shen Y, Chen J, Song Y, Chen S, Song Y, Wang L (2017) Sens Actuators B 239:890-897

74. Neumann B, Kielb P, Rustam L, Fischer A, Weidinger IM, Wollenberger U (2017) ChemElectroChem 4:913-919

75. Korri-Youssoufi H, Desbenoit N, Ricoux R, Mahy JP, Lecomte S (2008) Mater Sci Eng C 28:855-860

76. Ramanavicius A, Kausaite A, Ramanaviciene A (2005) Biosens Bioelectron 20:1962-1967

77. Ramanavicius A, Ramanaviciene A (2009) Fuel Cells 9:25-36

78. Ramanavicius A, Kausaite A, Ramanaviciene A (2008) Biosens Bioelectron 24:761-766 